\title{
NONNEGATIVE POLYNOMIALS, SUMS OF SQUARES AND THE MOMENT PROBLEM
}

\author{
ABHISHEK BHARDWAJ \\ (Received 7 January 2021; first published online 19 February 2021)
}

2020 Mathematics subject classification: primary 11E25; secondary 13J30, 15A45, 44A60, 46L07, 47A57, 81P45, 90C22.

Keywords and phrases: completely positive maps, moment problem, moments, polynomial optimisation, positive maps, quantum information, sums of squares.

Optimisation is one of the most widely applicable branches of mathematics across science. It is ubiquitous in statistics, biology, cosmology, engineering and computer science. In this thesis we study aspects of the special subclass of polynomial optimisation problems: given polynomials $p, g_{1}, \ldots, g_{k} \in \mathbb{R}\left[x_{1}, \ldots, x_{n}\right] \equiv \mathbb{R}[x]$, compute

$$
p^{\min }=\min _{x \in \mathbb{R}^{n}} p(x)
$$

such that $g_{1}(x) \geq 0, \ldots, g_{k}(x) \geq 0$.

In other words, find the minimum (or more generally the infimum) of the polynomial $p$ over the semi-algebraic set $K=\left\{x \in \mathbb{R}^{n}: g_{1}(x) \geq 0, \ldots, g_{k}(x) \geq 0\right\}$, which is the nonnegativity set of the polynomials $g_{1}, \ldots, g_{k}$. Many problems from different areas of mathematics can be formulated in this way; some notable examples are the max-cut problem, testing matrix copositivity and the stable set problem.

In the first part of this thesis, we study a polynomial optimisation problem which arises when solving the separability problem in quantum information theory, which asks to determine if a given quantum state $\rho$ is entangled. Letting $M_{n}(\mathbb{R})$ denote the space of $n \times n$ matrices, the most general criterion for answering this question is as follows.

CRITERION 1. A quantum state $\rho \in M_{n m}(\mathbb{R})$ is entangled if there is a positive map which is not completely positive (PNCP map)

$$
\Phi: M_{m}(\mathbb{R}) \rightarrow M_{k}(\mathbb{R})
$$

Thesis submitted to the Australian National University in February 2020; degree approved on 27 July 2020; supervisors Martin Helmer and Igor Klep.

(C) 2021 Australian Mathematical Publishing Association Inc. 
such that

$$
(I \otimes \Phi)(\rho) \nsucceq 0,
$$

where I is the identity matrix of appropriate size.

We consider a real algebraic geometry reformulation of the separability problem. It is easy to see that the map $\Phi$ gives rise to a biquadratic, bihomogeneous polynomial $p_{\Phi} \in \mathbb{R}[x, y]$ with

$$
p_{\Phi}(x, y)=y^{T} \Phi\left(x^{T} x\right) y .
$$

Moreover, the following bijective correspondence is a well-known result (see [6]).

THEOREM 2. The map $\Phi$ is positive if and only if $p_{\Phi}$ is nonnegative on $\mathbb{R}^{n+m}$, and $\Phi$ is completely positive if and only if $p_{\Phi}$ is a sum of squares (SOS) on $\mathbb{R}^{n+m}$.

In their recent work, Blekherman et al. [4] showed how to construct (random) nonnegative polynomials, over varieties of nonminimal degrees, which are not sums of squares. They further refined this process into an algorithm [6, Algorithm 1] to construct nonnegative biquadratic polynomials which are not sums of squares and have a carefully chosen set of zeros. As Criterion 1 relies on PNCP maps, Algorithm 1 gives an approach to solving the separability problem.

The underlying optimisation problem in Algorithm 1 requires minimising a nonnegative polynomial. When we restrict to strictly positive polynomials, there are many methods to decompose a polynomial into a sum of squares which are guaranteed to work, and work well. On the contrary, when we consider nonnegative polynomials, many of these methods (while useful) start to become limited and require additional information on intricate algebraic objects, which, in most cases, is difficult to obtain. Hence, the construction of PNCP maps from Algorithm 1 requires a computationally suitable sum of squares relaxation for nonnegative polynomials.

Our focus is on the development of practical computational methods to address these problems. We review classical sums of squares relaxations and give a comparison of the computational complexities between some of the modern state-of-the-art relaxations. Using the insights gained from this analysis we develop a MATLAB package, PNCP (see https://bitbucket.org/Abhishek-B/pncp/), which is able to solve the separability problem in cases which were beyond the reach of previously existing software implementations.

In the second part of this thesis, we study the truncated tracial moment problem, which can be thought of as a dual problem to noncommutative ( $n c$ ) polynomial optimisation, a generalisation of the classical commutative $(\mathrm{cm})$ moment problem. Let $\langle X, Y\rangle$ denote the free monoid generated by $X$ and $Y$. Two words $v, w \in\langle X, Y\rangle$ are cyclically equivalent $(w \sim v)$ if there are words $u_{1}, u_{2} \in\langle X, Y\rangle$ such that $w=u_{1} u_{2}$ and $v=u_{2} u_{1}$. For a word $w \in\langle X, Y\rangle$, let $w^{*}$ be the reverse of $w$. A sequence of numbers $\left(\lambda_{w}\right)$ indexed by words $w \in\langle X, Y\rangle$ satisfying

$$
\lambda_{w}=\lambda_{v} \text { whenever } w \sim v, \quad \lambda_{w}=\lambda_{w^{*}} \text { for all } w
$$


and $\lambda_{\phi}=1$ is called a (normalised) tracial sequence. As an example, given $n \in \mathbb{N}$ and a positive probability measure $\mu$ on $\left(\mathbb{S R}^{n \times n}\right)^{2}$, the sequence given by

$$
\lambda_{w}:=\int \operatorname{tr}(w(A, B)) d \mu(A, B)
$$

is a tracial sequence. The truncated tracial moment problem is the converse of this: for which sequence $\lambda \equiv\left(\lambda_{w}\right)_{\leq k}$, where the length of $w$ is at most $k$, do there exist an $n \in \mathbb{N}$ and a positive probability measure $\mu$ on $\left(\mathbb{S R}^{n \times n}\right)^{2}$ such that

$$
\lambda_{w}=\int \operatorname{tr}(w(A, B)) d \mu(A, B) ?
$$

For the bivariate quartic tracial moment problem, the problem is well understood when the associated Hankel matrix (which has size $7 \times 7$ ) is positive definite [5], or positive semi-definite and of rank at most 4 [2].

Here we examine the Hankel matrix when it is of rank 5 or 6 . Our first result reduces the study of the truncated tracial moment problem on quadratic varieties to four canonical cases.

THEOREM 3 (Canonical reductions). Suppose that the matrix $\mathcal{M}_{N C}(2)$ is of rank 5 or 6. If $\lambda$ admits an nc measure, then we have the following results.

(1) If $\mathcal{M}_{N C}(2)$ is of rank 5, then it satisfies $\mathbb{X Y}+\mathbb{Y} \mathbb{X}=\mathbf{0}$ and one of the following relations:

(a) Basic relation 1: $\mathbb{X}^{2}+\mathbb{Y}^{2}=\mathbb{1}$;

(b) Basic relation 2: $\mathbb{Y}^{2}=\mathbb{1}$;

(c) Basic relation 3: $\mathbb{Y}^{2}-\mathbb{X}^{2}=\mathbb{1}$;

(d) Basic relation 4: $\mathbb{Y}^{2}=\mathbb{X}^{2}$.

(2) If $\mathcal{M}_{N C}(2)$ is of rank 6 , then it satisfies one of the following relations:

(a) Basic relation 1: $\mathbb{Y}^{2}=\mathbb{1}-\mathbb{X}^{2}$;

(b) Basic relation 2: $\mathbb{Y}^{2}=\mathbb{1}+\mathbb{X}^{2}$;

(c) Basic relation 3: $\mathbb{X} Y+\mathbb{Y} \mathbb{X}=\mathbf{0}$;

(d) Basic relation 4: $\mathbb{Y}^{2}=\mathbb{1}$.

Furthermore, in two out of the four rank- 6 cases, we reformulate the existence of a representing measure to a feasibility problem of three small linear matrix inequalities (LMI) and a rank constraint. The next theorem demonstrates this for one of the cases (see [1, Ch. 4] or [3] for a more detailed exposition).

THEOREM 4 (Rank 6 to LMI). Suppose that $\mathcal{M}_{N C}(2)$ is of rank 6 satisfying the relation $\mathbb{Y}^{2}=\mathbb{1}-\mathbb{X}^{2}$. Then $\lambda$ admits an $n c$ measure if and only if there exist $a, b, c, d, e \in \mathbb{R}$ such that:

(1) $L(a, b, c, d, e) \geq 0$;

(2) $\mathcal{M}_{N C}(2)-L(a, b, c, d, e) \geq 0$; 
(3) $\left(\mathcal{M}_{N C}(2)-L(a, b, c, d, e)\right)_{\{\mathbb{1}, \mathbb{X}, \mathbb{Y}, \mathbb{X} \mathbb{Y}\}}>0$;

(4) $\operatorname{rank}(L(a, b, c, d, e)) \leq \operatorname{card} \mathcal{V}_{L}$,

where $L(a, b, c, d, e)$ is the following linear matrix polynomial:

$$
\left(\begin{array}{ccccccc}
a & \lambda_{X} & \lambda_{Y} & b & c & c & a-b \\
\lambda_{X} & b & c & \lambda_{X^{3}} & \lambda_{X^{2} Y} & \lambda_{X^{2} Y} & \lambda_{X}-\lambda_{X^{3}} \\
\lambda_{Y} & c & a-b & \lambda_{X^{2} Y} & \lambda_{X}-\lambda_{X^{3}} & \lambda_{X}-\lambda_{X^{3}} & \lambda_{Y}-\lambda_{X^{2} Y} \\
b & \lambda_{X^{3}} & \lambda_{X^{2} Y} & d & e & e & b-d \\
c & \lambda_{X^{2} Y} & \lambda_{X}-\lambda_{X^{3}} & e & b-d & b-d & c-e \\
c & \lambda_{X^{2} Y} & \lambda_{X}-\lambda_{X^{3}} & e & b-d & b-d & c-e \\
a-b & \lambda_{X}-\lambda_{X^{3}} & \lambda_{Y}-\lambda_{X^{2} Y} & b-d & c-e & c-e & a-2 b+d
\end{array}\right)
$$

with $a, b, c, d, e \in \mathbb{R}$.

Our results significantly improve previous approaches to the bivariate quartic tracial moment problem; ordinarily, the search for a representing measure is carried out via flat extensions: however, this approach teems with numerical instabilities and for larger size problems quickly becomes computationally intractable. In comparison, the computational complexity of the linear matrix inequalities remains the same and these can be efficiently solved.

Finally, we also study the tracial moment problem on elliptic curves. Our first result of this study reduces the tracial moment problem on elliptic curves to the classical moment problem on elliptic curves in two out of the three cases.

THEOREM 5 (Tracial to commutative). Let $\mathcal{M}_{N C}(n)$ be a positive-semi-definite, recursively generated moment matrix which satisfies $\mathbb{Y}^{2}=\mathbb{X}^{3}+a \mathbb{X}+b \mathbb{1}$. If $a \geq 0$ and $\mathcal{M}_{N C}(n)$ has a representing measure $\mu$, then $\mathcal{M}_{N C}(n)$ is a commutative moment matrix and has a commutative representing measure $\mu_{c m}$.

Furthermore, for the classical moment problem on elliptic curves, we give sufficient conditions for a representing measure $\mu$ to exist.

Theorem 6 (Commutative flat extension). Let $n \geq 3$. Suppose that $\mathcal{M}_{N C}(n)$ is positive and elliptic-pure. A flat extension $\mathcal{M}_{N C}(n+1)$ of $\mathcal{M}_{N C}(n)$ exists if the quartic polynomial $Q(\theta)$ has a real root.

\section{References}

[1] A. Bhardwaj, Trace Positive, Non-commutative Polynomials and the Truncated Moment Problem, Master's Thesis, University of Auckland, 2016, https://researchspace.auckland.ac.nz.

[2] A. Bhardwaj, Non-negative Polynomials, Sums of Squares \& the Moment Problem, PhD Thesis, The Australian National University, Canberra, 2020, http://openresearch-repository.anu.edu.au.

[3] A. Bhardwaj and A. Zalar, 'The singular bivariate tracial moment problem', Complex Anal. Oper. Theory 12 (2018), 1057-1142.

[4] G. Blekherman, G. Smith and M. Velasco, 'Sums of squares and varieties of minimal degree', J. Amer. Math. Soc. 29(3) (2016), 893-913. 
[5] S. Burgdorf and I. Klep, 'Trace-positive polynomials and the quartic tracial moment problem', $C$. $R$. Math. 348(13-14) (2010), 721-726.

[6] I. Klep, S. McCullough, K. Šivic and A. Zalar, 'There are many more positive maps than completely positive maps', Int. Math. Res. Not. 2019(11) (2019), 3313-3375.

\section{ABHISHEK BHARDWAJ,}

Laboratoire d'analyse et d'architecture des systèmes (LAAS),

Centre National de la Recherche Scientifique, 31400 Toulouse, France

e-mail: Abhishek.Bhardwaj.Maths@gmail.com 